„Offensive Zukunft Bayern" aus dem Allgemeinen Umweltfond (Anschubfinanzierung 5 Mio. DM). Die Einrichtung ist organisatorisch der Klinik und Poliklinik für Dermatologie und Allergologie am Biederstein (Prof. Johannes Ring) der TU München angegliedert.

In dem neu zu errichtenden ,Zentrum Allergie und Umwelt" sollen im interdisziplinären Ansatz naturwissenschaftliche Grundlagenforschung,

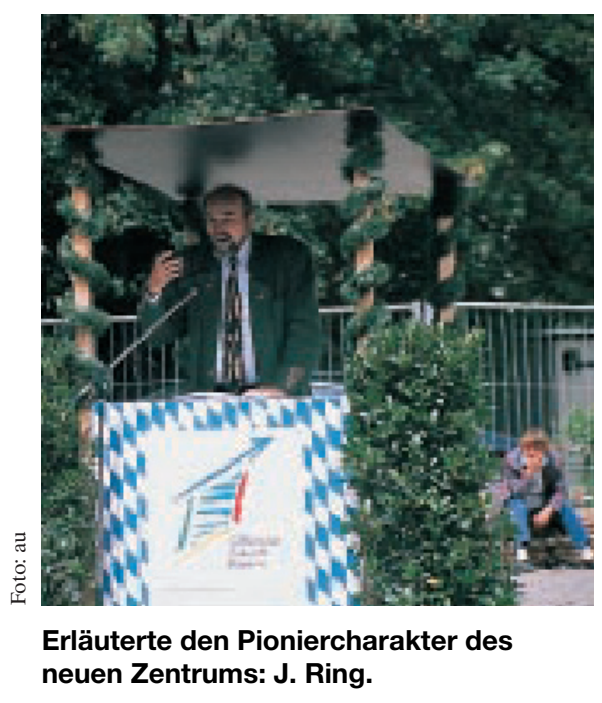

biomedizinisch und klinisch-experimentelle Forschung sowie klinisch angewandte Forschung mit Beratung und Versorgung angesiedelt werden. Das Forschungsziel ist die Bewertung der Einflüsse von Umweltfaktoren auf die Entstehung, Auslösung und Unterhaltung allergischer Erkrankungen. Daraus sollen neue Konzepte zu Diagnose, Therapie und Prävention erarbeitet werden.

Das „Zentrum Allergie und Umwelt" solle zwischen Grundlagenwissenschaften, Klinik und Umweltforschung angesiedelt sein und die im Großraum München an AllergieForschung interessierten Kräfte bündeln, erklärte J. Ring. „Die Verbindung von Allergie- und Umweltforschung einschließlich ihrer Anwendung in Versorgung, Beratung und Prävention ist neu. Es gibt weltweit keine vergleichbare Einrichtung."

Kontakt: Prof. Dr. Dr. Johannes Ring, Tel. 089/41403170, Fax 089/41 403553, e-mail: johannes.ring@lrz.tu-muenchen.de

Allergische Erkrankungen sind weltweit auf dem Vormarsch. Trotz verbesserter Diagnostik und Therapie, vorbeugender Aufklärungsarbeit (z.B. Pollenflugvorhersage) und öffentlicher Aufmerksamkeit gibt es auch in Deutschland noch viel zu tun, um die Situation von Allergikern zu verbessern. Der Ärzteverband Deutscher Allergologen hat sich deshalb entschlossen, durch kontinuierliche Presse- und Öffentlichkeitsarbeit auf bestehende Probleme hinzuweisen. Im folgenden und in weiteren Ausgaben des ALLERGO JOURNAL finden Sie zu Ihrer Information Auszüge aus Pressemeldungen (Quellenangabe „ÄDA-Pressekontakt"), die bewußt über die Grenzen der Fachpresse hinaus verteilt und so einer breiten Basis zugänglich gemacht werden.

\title{
Allergie-Impfung: Therapiestandard im 21. Jahrhundert
} Erfolge auch bei der Behandlung von allergischen Kindern

er Schlußtag des 17. Kongresses der Europäischen Akademie für Allergologie und klinische Immunologie in Birmingham stand noch einmal ganz im Zeichen der AllergieImpfung (Hyposensibilisierung). Vor 3000 Experten aus 70 Nationen stellte H.-J. Malling, Kopenhagen, eine zusammenfassende Bewertung von 50 wissenschaftlichen Arbeiten über diese Therapieform vor. Das Ergebnis: Die Impfung beugt bei frühzeitiger Anwendung einer Verschlimmerung allergischer Erkrankungen vor, verbessert die Lebensqualität, reduziert stark den Verbrauch von Begleitmedikationen und damit auch die Langzeitkosten im Gesundheitsbudget.

„Die Ergebnisse sind eindeutig. Die größten Erfolge sind derzeitig bei der Impfung mit Pollenextrakten zu verzeichnen. Darüber hinaus sind Hyposensibilisierungen bei Hausstaubmilben- und Schimmelpilzallergien sinnvoll," unterstrich Malling.

Neue Erkenntnisse wurden in Birmingham auch bei der Behandlung von Kindern präsentiert. AllergieSpezialisten aus Schweden, Finnland,
Deutschland, Österreich und Dänemark analysierten die Bedeutung der Allergie-Impfung bei Jugendlichen. In der sog. PAT-Studie (Preventive Allergy Treatment) wurden $210 \mathrm{Kin}$ der mit saisonalem Heuschnupfen einbezogen und auf die Entwicklung von Asthma untersucht. Kinder, die über drei Jahre eine Allergie-Impfung erhalten hatten, trugen ein dreifach geringeres Risiko, an Asthma zu erkranken, als lediglich symptomatisch behandelte Kinder.

Das Qualitätsmanagement der Hyposensibilisierung ist die Aufgabe des 21. Jahrhunderts. Die Allergologen forderten in Birmingham eine patientenfreundlichere Form der Impfung. Außerdem sind patientenorientierte Allergie-Impfungszentren mit längeren Öffnungs- und ohne Wartezeiten dringend erforderlich. „Die AllergieImpfung hat eine vielversprechende Zukunft“, erläuterte Malling. „Sie sollte ergänzend zu der medikamentösen Therapie, nicht aber in Konkurrenz mit ihr, von erfahrenen AllergieSpezialisten angewendet werden."

ÄDA-Pressekontakt, Bodelschwinghstr. 17, D-22337 Hamburg. 